Email address: junwang@tongji.edu.cn (Jun Wang)

Preprint submitted to Elsevier

Keywords: wind turbine, wake flow, particle random walk

\section{Introduction}

\title{
Simulating the wake flow effect of wind turbines on velocity and turbulence using particle random walk method
}

\author{
MengXuan Song ${ }^{\mathrm{a}}$, BingHeng $\mathrm{Wu}^{\mathrm{b}}$, Kai Chen ${ }^{\mathrm{c}}$, Xing Zhang ${ }^{\mathrm{b}}$, Jun Wang ${ }^{\mathrm{a}}$ \\ ${ }^{a}$ Department of Control Science and Engineering, Tongji University, Shanghai 201804, P. \\ R. China \\ ${ }^{b}$ Key Laboratory for Thermal Science and Power Engineering of Ministry of Education, \\ Department of Engineering Mechanics, Tsinghua University, Beijing 100084, P. R. China \\ ${ }^{c}$ Key Laboratory of Enhanced Heat Transfer and Energy Conservation of the Ministry of \\ Education, School of Chemistry and Chemical Engineering, South China University of \\ Technology, Guangzhou 510640, Guangdong, P. R. China
}

\begin{abstract}
This paper presents a novel way of simulating the effect of velocity decay and turbulence of wind turbine's wake flow. By decoupling the solving of wake flow from that of the velocity field, the proposed model treats the wake flow intensity as a kind of convective and diffusive virtual matter. The particle random walk method is utilized to simulate the motion of the virtual matter. Comparing to the existing linear model for turbine wake flow, the proposed model can predict the distributions of velocity decay and turbulence of wake flow in a non-uniform flow field above complex terrain. Experimental data from wind tunnel and real wind farm is used to validate the model, demonstrating its effectiveness on estimating the velocity decay and the turbulence intensity, and additionally, the power yield of a wind farm. The model proposed in this paper can be integrated into algorithms for numerical assessment and micro-siting optimization of wind farms on complex terrains.
\end{abstract}

Using horizontal-axis wind turbines for wind power generation is one of the most mature technologies of utilization of clean and renewable energies. Besides the efficiencies of the wind turbines and the power grid, wind turbine positioning is remarkably influential to the amount of power yield of a wind farm. Since wind wake reduces wind speed and increases turbulence in the downstream region, irrational layout of wind turbines within a wind farm will lead to high interactions among the turbines, causing unnecessary power loss.

Multiple intelligent algorithms have been studied on solving the wind turbine positioning problem. Mosetti et al. [1] introduced Genetic Algorithm (GA) to

(C) 2016. This manuscript version is made available under the Elsevier user license 
this problem, using a $10 \times 10$ Cartesian mesh. After them, various studies have been reported in developing new approaches for optimization of wind turbine positioning, such as using triangular-shaped grids instead of Cartesian mesh [2], encoding the turbine layout into real number sequences instead of binary coding [3], adopting the Particle Swarm Optimization (PSO) algorithm [4], Monte Carlo algorithm [5] and greedy algorithm [6], etc.

In all optimization algorithms of wind turbine positioning, in order to search for the best layout for wind turbines, it is essential for the process of evaluating the layout's power yield to be run for a large number of times, leading to a big challenge in developing an appropriate model for wake flow estimation. The wake flow estimation model must run fast, otherwise the optimization process will cost unacceptable time to obtain an optimized solution.

The most widely used model for wake flow estimation is the linear model proposed by Jensen [7]. It treats the wake affected region as a conical area, inside which the amount of velocity decay is unrelated to the radial position. Similar but more complicated models are proposed by Larsen [8] and Ishihara et al. [9] respectively. Although more factors have been taken under consideration in their improved models, all the parameters of the wake flow are still determined only by the flow states at the turbine. Downstream variations of airflow are unable to influence the behavior and characteristics of the wake flow. These simplified models are limited for flat terrain only.

On the other hand, researchers in the field of fluid mechanics performed detailed numerical simulation using Computational Fluid Dynamics (CFD) [10, $11,12,13,14]$, and experimented on sophisticated wind tunnel measurements of wind turbine wake flow $[15,16]$. Their results describe the nature of wake flow accurately under many circumstances, providing better understanding on how multiple wake flows interact and overlap with each other. However, in the optimization of wind turbine positioning in a wind farm, any optimization algorithm requires multiple times of wake flow evaluation for a large quantity of different turbine layouts. An ordinary iteration of solving the flow field around a wind turbine may takes hours to converge. Time cost on simulating wake flow behaviors for multiple wind turbines inside a wind farm for multiple different layouts is even more unacceptable. It is not practical to integrate CFD directly into optimization approaches for wind turbine positioning.

A wake flow model using the particle random walk technique has been previously proposed [17]. This model treats the wake flow effect as convective and diffusive virtual particles carried by background airflow. As the background flow field can be obtained from CFD calculation, wake flow predicted by the particle model will bend according to local downstream airflow, for which it is adaptable for non-uniform flow field above complex terrain. Additionally, since the background flow field needs to be evaluated for only once, the model runs fast enough to be integrated with optimizations. Subsequent studies $[18,19,20]$ demonstrated the usefulness of this model. Nevertheless, the relationship between the particles' diffusive motions and the incoming wind speed was not considered, the attenuation process of the particles was not clearly defined, and the overlapping phenomenon of multiple wake flows was not comprehensively 
discussed.

This paper presents the advanced version of the wake flow model using particle random walk technique. The limitations mentioned above are overcome, and additionally, a model to estimate turbulence intensity within wake regions is developed. The remainder of the paper is organized as follows. Section 2 describes the linear wake model, which will be used in comparisons in the other sections. Section 3 overviews the previous version of the particle model and presents the improvements. Section 4 presents the model for turbulence estimation. Results are validated by experimental data in a wind tunnel and measurements on a real wind farm. Section 5 concludes the paper.

\section{Overview of the linear wake model}

The linear wake model was developed by Jensen et al. [7, 21]. Gonzalez et al. [22] considered that in most situations, wake flow regions do not cover entire rotors of the downstream turbines, and the model was modified by adding a term representing the overlapped area of the wake region and the affected turbine rotor. Therefore, the modified model is used in the wind farm evaluation in Section 3.4. In sections except Section 3.4, since our purpose is to calculate the velocities at certain coordinates within the field, not the wake effect to other turbines, the original model is used.

For the original model, the wake affected region of a single wind turbine is sketched in Figure 1. The wake flow is originated at the position of the turbine rotor, and develops in a conical shape. $x$ indicates the distance from the turbine hub to a downstream position, projected to the streamwise direction. The radius of the wake affected region at distance $x$ is

$$
r_{\mathrm{w}}=\alpha x+r_{\mathrm{r}},
$$

where $r_{\mathrm{r}}$ is the radius of the turbine rotor (including blades), $\alpha$ is the entrainment constant calculated by

$$
\alpha=\frac{0.5}{\log \left(h / z_{0}\right)},
$$

where $h$ is the height of the turbine hub to the ground, $z_{0}$ is the ground roughness length. Let $u$ be the speed of the incoming wind. The wind speed outside the wake region remains unchanged, while the wind speed inside the wake region is calculated through

$$
u^{\prime}=u\left[1-\frac{2 a}{\left(1+\alpha x / r_{1}\right)^{2}}\right],
$$

where $a$ and $r_{1}$ are calculated by

$$
a=\frac{1-\sqrt{1-C_{\mathrm{T}}}}{2}, \quad r_{1}=r_{\mathrm{r}} \sqrt{\frac{1-a}{1-2 a}},
$$

where $C_{\mathrm{T}}$ is the thrust coefficient of the wind turbine. 
For scenario with multiple turbines, the first step is to calculate the velocity in the wake region for each turbine separately using Equation 3, whose result is denoted as $u_{i}^{\prime}$, where $i$ stands for the $i$ th turbine. Then the overall velocity in the wake region is

$$
u^{\prime}=u-\sqrt{\sum_{i=1}^{N}\left(u-u_{i}^{\prime}\right)^{2}}
$$

where $N$ is the total number of turbines.

For the modified model, Equation 5 is modified into

$$
u^{\prime}=u-\sqrt{\sum_{i=1}^{N}\left[\frac{A_{i}}{\pi r_{\mathrm{r}}^{2}}\left(u-u_{i}^{\prime}\right)^{2}\right]},
$$

where $r_{\mathrm{r}}$ is rotor radius of the turbine, $A_{i}$ is the overlapped area of the wake region generated by the $i$ th turbine and the turbine being affected. Figure 2 plots the wake region and the turbine rotor being affected as a projection onto the plane of the turbine rotor. $O_{\mathrm{T}}$ is the center of the turbine being affected, while $O_{\mathrm{w}, i}$ is the center of the wake region generated by the $i$ th turbine. $r_{\mathrm{w}, i}$ is the radius of the wake affected region of the $i$ th turbine, calculated by Equation 1. According to basic geometry, the overlapped area of the two circles is

$$
A_{i}= \begin{cases}\pi r_{\mathrm{r}}^{2}, & d_{i} \leq r_{\mathrm{w}, i}-r_{\mathrm{r}} \\ \frac{1}{2} r_{\mathrm{r}}^{2}\left(\theta_{1}-\sin \theta_{1}\right)+\frac{1}{2} r_{\mathrm{w}, i}^{2}\left(\theta_{2}-\sin \theta_{2}\right), & r_{\mathrm{w}, i}-r_{\mathrm{r}}<d_{i}<r_{\mathrm{w}, i}+r_{\mathrm{r}} \\ 0, & d_{i} \geq r_{\mathrm{w}, i}+r_{\mathrm{r}}\end{cases}
$$

where

$$
\begin{aligned}
& \theta_{1}=2 \arccos \frac{r_{\mathrm{r}}^{2}+d_{i}^{2}-r_{\mathrm{w}, i}^{2}}{2 r_{\mathrm{r}} d_{i}}, \\
& \theta_{2}=2 \arccos \frac{r_{\mathrm{w}, i}^{2}+d_{i}^{2}-r_{\mathrm{r}}^{2}}{2 r_{\mathrm{w}, i} d_{i}} .
\end{aligned}
$$

\section{The present model for velocity}

One of the primary effects caused by wake flow is velocity decay in the downstream region of a wind turbine. The common concept of the widely used wake models is to decouple the calculation of velocity decay from the background flow field, as mentioned in the introduction. In other words, a background flow field without any influence of wake flow is pre-obtained by other means, and the amount of velocity decay at each position is superposed onto the background flow field, producing the wake-influenced velocity field. The model proposed in this paper imitates this similar idea of decoupling, but calculates the amount of velocity decay in a more accurate way. 


\subsection{Velocity decay and random walk particles}

The particle model, as described in a previous paper [17], assumed that the behavior of the ratio of velocity decay acts like a kind of virtual convective and diffusive matter. The matter is produced by rotating turbine blades, and carried to downstream regions by airflow in the background flow field. Chen et al. demonstrated that this phenomenon of scalar transportation can be numerically calculated through particle random walk method [23]. Using this technique, the calculation of velocity decay is performed in two steps. First, multiple particles are generated in the area of the rotating blades of each turbine, and the convective and diffusive motions of the particles are simulated for a certain length of time period. During the simulation, the number of particles passing through each concerned region is counted. Second, the counted numbers are transformed into magnitudes of velocity decay, which are then superposed to the background flow field, producing the final result. The details are explained as follows.

In every time step of the simulation, particles are generated randomly with uniform distribution within the circular area. For each newly generated particle, its radial and angular position with respect to the rotor center is calculated by

$$
r=\sqrt{R_{1}} r_{\mathrm{r}}, \quad \alpha=2 \pi R_{2},
$$

where $r_{\mathrm{r}}$ is the rotor radius of the turbine (including the blades), $R_{1}$ and $R_{2}$ are two independent random numbers with uniform distribution on $[0,1)$. Then the birthplace of the particle is calculated by

$$
\mathbf{x}=\mathbf{x}_{\mathrm{T}}+\left(\begin{array}{c}
0 \\
r \cos \alpha \\
r \sin \alpha
\end{array}\right),
$$

where $\mathbf{X}_{\mathrm{T}}$ is the coordinate of the center of the turbine rotor.

Within each time step of the simulation, the displacement of a particle consists of its convective displacement and its diffusive displacement:

$$
\Delta x_{i}=\Delta x_{i, c}+\Delta x_{i, d}
$$

where its convective displacement is identical to the motion of the local fluid, which is

$$
\Delta x_{i, c}=u_{i} \Delta t
$$

while the diffusive displacement is approximated by a Gaussian random number. Box and Muller suggested that a Gaussian random number can be generated by $\sqrt{-2 \log R_{3}} \cos \left(2 \pi R_{4}\right)$, where $R_{3}$ and $R_{4}$ are two independent random numbers with uniform distribution on $[0,1)[24]$. Einstein proposed that in Brownian movement, the average length of the square of diffusive displacement is proportional to time [25], that is $\overline{x_{i, d}^{2}} \propto \Delta t$. Then the diffusive displacement is calculated by

$$
\Delta x_{i, d}=\sigma \sqrt{-2 \Delta t \log R_{3}} \cos \left(2 \pi R_{4}\right),
$$


where $\sigma$ characterizes the strength of the diffusive motion, $\Delta t$ is the length of the time step, and $R_{3}$ and $R_{4}$ are two independent random number with uniform distribution on $[0,1)$.

Former models of turbine wake flow imply that the shape of the wake affected region is unrelated to the incoming velocity and the scale of the turbine. Therefore we choose $\sigma=\sigma_{d} \sqrt{\|\mathbf{u}\| r_{\mathrm{r}}}$ (where $\sigma_{d}$ is a constant) in order to make the scale of $\Delta x_{i, d} / \Delta x_{i, c}$ unrelated to the velocity and the rotor radius. The final formula to calculate the overall motion of a particle within a simulation time step is

$$
\Delta x_{i}=u_{i} \Delta t+\sigma_{d} \sqrt{-2\|\mathbf{u}\| r_{\mathrm{r}} \Delta t \log R_{3}} \cos \left(2 \pi R_{4}\right) .
$$

A regular attenuation process is added to the particles to compensate for the deviations caused by the decoupling approximation. A half-life period is assigned to each particle, denoted as $\tau$. Therefore in each time step of simulation, the probability of each particle to vanish is

$$
\gamma(\Delta t)=1-\left(\frac{1}{2}\right)^{\Delta t / \tau} .
$$

After a certain length of simulation is performed, the counted number of particles per unit volume in each concerned region (denoted as $n(\mathbf{x})$ ) is transformed into particle concentration through

$$
c(\mathbf{x})=\frac{n(\mathbf{x}) u_{0}}{p}
$$

where $u_{0}$ is the wind speed in the background flow field at the position of the center of the turbine rotor, and $p$ is the number of particles generated per unit area and unit time span. With the particle concentration calculated, the wind velocity with velocity decay superposed is obtained by

$$
u^{\prime}(\mathbf{x})=u(\mathbf{x})(1-\beta c(\mathbf{x})),
$$

where $\beta$ is a constant. Theoretically $c(\mathbf{x})$ is 1 near the rotor center in the downstream direction [23].

On the other hand, the traditional linear model calculates the wake-influenced velocity through Equation 19.

$$
u^{\prime}(x)=u(x)\left[\frac{2 a}{\left(1+\alpha x / r_{1}\right)^{2}}\right],
$$

where $x$ is the distance originated from the rotor center towards the downstream direction along the rotor axis, $\alpha$ is the entrainment constant, $r_{1}$ is a constant related to the rotor radius, and $a$ is the axial induction factor of the turbine, which has the following relationship with its thrust coefficient $C_{\mathrm{T}}$.

$$
C_{\mathrm{T}}=4 a(1-a) .
$$

In order to maintain consistency with the linear model at $x=0$, we can deduce that $\beta=2 a$, leaving $\sigma_{d}$ and $\tau$ to be determined. 


\subsection{Calibration for a single turbine}

$\sigma_{d}$ and $\tau$ can differ for different types of turbines. In the previous study, we took the Nibe-B $630 \mathrm{~kW}$ wind turbine as an example to calibrate the particle model [17]. This type of turbine has its rotor diameter at $40 \mathrm{~m}$ (including blades), and its hub height at $45 \mathrm{~m}$. However, in the previous study, the wake shape is not unrelated with velocity and rotor radius, and the half-life attenuation process is not revealed. Calibration in the previous study concludes that $\sigma=0.3$ and $\gamma=0.005$ are proper for this type of turbine, with $u_{0}=8.5 \mathrm{~m} / \mathrm{s}$ and $\Delta t=1 \mathrm{~s}$. Therefore, according to the definitions of $\sigma$ and $\gamma$ in the previous section, it can be deduced that

$$
\sigma_{d} \approx 0.23, \quad \tau \approx 138 \mathrm{~s} .
$$

The results of the calibration on $u_{0}=8.5 \mathrm{~m} / \mathrm{s}$ are plotted in Figures 3,4 and 5 , where the horizontal axis is the radial position normalized by the rotor diameter, and the vertical axis is the wind speed normalized by the incoming wind speed. $X$ is the streamwise coordinate, and $D$ is the diameter of the turbine rotor. Experimental data is provided by Taylor et al. [26]

\subsection{Wake overlap for multiple turbines}

In a wind farm with multiple wind turbines, particles generated by each turbine are simulated and counted separately. Denote the particle concentration at position $\mathbf{x}$ caused by the $i$ th turbine as $c_{i}(\mathbf{x})$. In the previous study of the particle model [17], the overall particle concentration referred by Equation 18 is the sum of all concentrations:

$$
c(\mathbf{x})=\sum_{i=1}^{N} c_{i}(\mathbf{x}),
$$

where $N$ is the number of turbines within the wind farm. This manner of wake overlapping may lead to high values of $c(\mathbf{x})$ and low values of $u^{\prime}(\mathbf{x})$, which is not like the real situation that happens in a real wake overlapped region.

In the present study, the overall particle concentration is simply assigned as

$$
c(\mathbf{x})=\max _{i=1,2, \cdots, N} c_{i}(\mathbf{x}) .
$$

Using this way of wake overlapping, wake flow generated by each turbine is calculated separately, and at any position $\mathbf{x}$, only the concentration of the strongest wake flow is considered to be the overlapped value.

Experimental data obtained by Chamorro et al. [15] is used to validate the particle model. In their study, 25 miniature turbines are placed in a staggered pattern inside a wind tunnel, as plotted in Figure 6, where wind blows along the positive direction of the $X$-axis. The rotor diameter (including blades) is $D=128 \mathrm{~mm}$ and the height of the hub is $h=104 \mathrm{~mm}$. Column spacing and row spacing of the layout are $4 D$ and $5 D$ respectively. Surface roughness is $z_{0}=0.05 \mathrm{~mm}$. 
The experimental data contains normalized measured velocity along the mainstream direction ( $X$ direction), in the 2nd column of turbines $(Y=2 D=$ $0.256 \mathrm{~m})$, at the heights of hub $(Z=h=0.104 \mathrm{~m})$, bottom tip $(Z=h-D / 2=$ $0.04 \mathrm{~m})$ and top tip $(Z=h+D / 2=0.168 \mathrm{~m})$ respectively.

The incoming velocity profile is specified by the logarithmic law:

$$
u(z)=\frac{\ln \left(z / z_{0}\right)}{\ln \left(h / z_{0}\right)} .
$$

The characteristics of the miniature turbine are different from those of the turbine used in the calibration in the previous section, and its thrust coefficient is unknown. Among the 2nd column of turbines, which have been experimented on, the region marked by blue dashed line in Figure 6 is the region with minimum wake overlapping. Measured velocities in this region are used to calibrate for thrust coefficient $C_{\mathrm{T}}$. Different values of $C_{\mathrm{T}}$ produce different velocity discrepancies between the calculated result and the experimental data within this region, as listed in Table 1. According to the result, it can be obtained that the best fit occurs when $C_{\mathrm{T}}=0.59$, with discrepancy at $4.6674 \%$.

With all the coefficients in the particle model determined, the model is applied to calculate the velocity distributions along the $X$-axis at $Z=0.104 \mathrm{~m}$, $0.168 \mathrm{~m}$ and $0.04 \mathrm{~m}$ respectively for the $2 \mathrm{nd}$ column of turbines. Results are plotted in Figures 7, 8 and 9, where the vertical coordinate is the streamwise velocity normalized by incoming wind speed at the height of the hub.

Results calculated using the linear wake model are also presented as black dotted lines for comparison. The algorithm of the linear model is briefly described in 2 .

It can be seen from the curves that the previous overlapping method overestimates the strength of the overlapped wake flow, especially in the further downstream region. The present overlapping method produces better results than the previous one. The errors for the hub, the rotor tip and the rotor bottom are all lower than the ones of the previous model, as listed in Table 2 and Table 3. And all the errors of the present model are below the errors of the linear model.

\subsection{Validation in real wind farm}

The particle model is then validated in a real wind farm at Wattle Point of Australia, whose geolocation is around 137.71E, 35.13S. 55 wind turbines of the type Vestas V82 (1.65 MW) were installed. The hub height is $68.5 \mathrm{~m}$ and the rotor diameter is $82 \mathrm{~m}$. Layout of the wind farm is sketched in Figure 11, where contours represent terrain altitude. Wind speed measurement is obtained from meteorological station ID022046, which is public data provided by the Australian government. Observed records for WSW wind direction between March 19 to April 15 of the year 2013 are picked for validation. Data of actual power yield is public data provided by Australian Energy Market Operator.

The presented particle model is a model that evaluates the influence of the wake flow to the downstream velocity. In order to validate the model with 
measured data of the power yield, the relationship between the incoming wind speed and the power output of the turbine is necessary. The theoretical power curve of Vestas V82 provided on its product brochure is plotted in Figure 10. Croonenbroeck et al. compared the theoretical power curve of Vestas V90 with the actual measured data from wind farms. They pointed out that the theoretical power curve differs from the real cases due to air density changes or the prudent estimation [27]. Therefore, in order to evaluate the power product of each turbine accurately, it is required to have a better model calibrated by using the actually measured wind speeds and power products on each turbine for various situations. Since the data with these details are not available for us at the present, we could only validate our model by using the theoretical power curve in this section, as a preliminary study. Further investigations are essential before the present model can be put into real application.

Figure 12 shows the relationship between the overall power output of the wind farm and the incoming wind speed for the picked records. The linear model overestimates the wake effect obviously, leading to lower estimation on the power output. The previous particle model is the most accurate one among the three models in the high speed range, and the present particle model acts better in the medium wind speed range. All the three models produce larger errors in the low wind speed range. The mean absolute error (MAE) of the linear model is $11000.86 \mathrm{~kW}$, while the ones of the previous particle model and the present particle model are $6854.598 \mathrm{~kW}$ and $6749.856 \mathrm{~kW}$ respectively. The root mean square errors (RMSE) of the linear model, the previous particle model and the present particle model are $13362.14 \mathrm{~kW}, 8832.453 \mathrm{~kW}$ and $8113.797 \mathrm{~kW}$, respectively.

\section{The present model for turbulence}

Although not exactly precise, the $k$ - $\varepsilon$ turbulence model implies that the behavior of turbulent energy can be approximated to be following the same law as a convective and diffusive scalar, and it has the same diffusion coefficient as the one for velocity or momentum in the wind farm scenarios [28]. Based on this phenomenon, idea similar to the model of velocity is implemented to simulate the turbulence intensity within the wake flow region of a wind turbine. Turbulence intensity is treated as a convective and diffusive virtual matter, and its developing process is simulated through the particle random walk method.

\subsection{Single turbine}

Differing from the velocity decay, most of the turbulent flow is generated by the blade tips, rather than being uniformly generated within the circular area. With consideration of this nature of turbulence generation, particles representing turbulence intensity (referred to turbulence particle for short below) are generated only on the perimeter of the circular area of the rotating blades. 
That is, the birthplace of the turbulence particle is

$$
\mathbf{x}=\mathbf{x}_{\mathrm{T}}+\left(\begin{array}{c}
0 \\
r_{\mathrm{r}} \cos \alpha \\
r_{\mathrm{r}} \sin \alpha
\end{array}\right),
$$

where $\mathbf{X}_{\mathrm{T}}$ is the position of the center of the turbine rotor, $r_{\mathrm{r}}$ is the radius of the turbine rotor (including blades), and $\alpha$ is a random angle uniformly distributed within $[0,2 \pi)$.

The rest of the simulation steps are the same as the ones for the velocity model. With the concentration of the particles obtained, turbulence intensity is calculated through the linear transformation:

$$
I(\mathbf{x})=\zeta \cdot c(\mathbf{x})+I_{0},
$$

where $\zeta$ and $I_{0}$ are transformation coefficients that need to be determined.

As mentioned before, the diffusive motion of the turbulence particles is with the same strength as the one for the velocity model, but its strength of attenuation differs, leaving its half-life period $\tau$ to be determined. Calibration for $\tau$ is also done using the experimental data from Taylor et al. [26]. For any value of $\tau$, best values of $\zeta$ and $I_{0}$ can be found to achieve the minimum discrepancy between the simulated results and the experimental data, using the Successive Quadratic Programming (SQP) algorithm. Different values of $\tau$ produce different minimum discrepancies. For the Nibe-B wind turbine, some of the results are listed in Table 4 . Then the best value of $\tau$ can be chosen to be the one that produces the overall minimum discrepancy, which is

$$
\tau=31 \mathrm{~s}, \quad \zeta=0.3373, \quad I_{0}=0.0784 .
$$

Fitted results are plotted in Figures 13 and 14. Discrepancies between our simulated results and the experimental data along the streamwise positions and the radial positions are 0.0057 and 0.0054 respectively.

\subsection{Multiple turbines}

Experiments done by Chamorro et al. discover that the turbulent flow generated by turbines in a staggered layout spreads more extensively upwards than downwards [15]. In order to compensate for this phenomenon in the present model, the birthplace of turbulence particles is amended from Equation 25 into

$$
\mathbf{x}=\mathbf{x}_{\mathrm{T}}+\left(\begin{array}{c}
0 \\
\pm r_{\mathrm{r}} \sqrt{1-R_{z}^{2}} \\
r_{\mathrm{r}} R_{z}
\end{array}\right)
$$

where

$$
R_{z}=2\left(\frac{1}{2} \sin \alpha+\frac{1}{2}\right)^{m}-1 .
$$


$m$ is an adjustable coefficient between 0 and 1 , having the ability of concentrating the birthplaces of turbulence particles towards the top of the circular area of the rotating blades.

Since the particles are generated along the perimeter of the circular area rather than inside it, particle concentration can be extremely high near the birthplace. Therefore, simulated data points within $1 D$ in the downstream direction of each turbine are discarded.

According to Equation 26, $I_{0}$ is the turbulence intensity where no turbulence particle exists. So measured data before the most upstream turbine in the measured column is used to determine the values of $I_{0}$, which are listed in the row labeled "Measured incoming flow" in Table 5. Similarly to the model for velocity, measured data at positions marked by the blue dashed line in Figure 6 is used to calibrate for $m$ and $\zeta$. It obtains that the best fit occurs when $m=0.15$ and $\zeta=0.0651$.

With the coefficients determined, the present model is applied to calculate the distribution of turbulence intensity along the mainstream direction at $Z=0.168 \mathrm{~m}, 0.104 \mathrm{~m}$ and $0.04 \mathrm{~m}$ respectively. Comparisons with Chamorro's experimental data are plotted in Figures 15, 16 and 17.

It can be noticed from Figure 16 that, even when simulated data points within $1 D$ in the downstream direction of each turbine are discarded, sharp peaks still exist after each turbine's top tip. The reason is that the generation process of the turbulence particles and that of the real turbulent flow is not exactly the same after all. Nevertheless, the present model is aimed at providing a fast yet accurate model for wind farm numerical assessment and micro-siting optimization, errors in the near-wake regions are irrelevant.

The errors are listed in Table 5. The relative errors of the present model are around $7.6 \%$ to $12.5 \%$. Considering that the turbulent phenomenon is a rather complicated problem even for the field of fluid mechanics, the present model developed for fast assessment and optimization of wind farms can be acceptable.

\section{Conclusions}

This paper presented a fast model to calculate velocity decay and turbulence of the wake flow of wind turbines. The most significant improvement comparing to existing traditional models is that the wake flow simulated by the proposed model can be distorted by downstream local airflow, making it capable to predict wake flow's intensity and shape in non-uniform flow fields above complex terrains. The proposed wake flow model simulates the wake flow decoupled from the velocity field, and utilizes the meshless particle random walk method. It has balanced performance on efficiency and accuracy.

Comparisons with measurements in a wind tunnel validate the effectiveness of the proposed wake flow model. The maximum MAE of the dimensionless velocity is lower than 0.06 , and the maximum relative error of the turbulence intensity is lower than $13 \%$. Comparison with wind farm data proves that the 
present model has higher agreement with experiments than the widely used linear model on real terrain. The relative error of the predicted power yield with respect to the total capacity drops from $12.1 \%$ to $7.4 \%$.

It is also notable that multiple improvements have been achieved since the previous version of the particle model: (1) adapted for wind turbines with different sizes; (2) has a more explicit definition on the attenuation process of wake particles; (3) overlapping of the velocity decay has been improved to have better agreement with the experimental data; (4) simulation of the turbulence intensity of the wake flow is capable using the same particle random walk technique.

\section{Acknowledgment}

This research is supported by the Program for Young Excellent Talents in Tongji University (2014KJ025), the National Science Foundation of China (51406136), and the International Science and Technology Cooperation Program of China (2011DFG13020).

\section{References}

[1] G. Mosetti, C. Poloni, B. Diviacco, Optimization of wind turbine positioning in large windfarms by means of a genetic algorithm, Journal of Wind Engineering and Industrial Aerodynamics 51 (1994) 105-116.

[2] X. Li, J. Wang, X. Zhang, Equilateral-triangle mesh for optimal micrositing of wind farms, in: 14th WSEAS International Conference on Computers, Corfu, Greece, 187-195, 2010.

[3] C. Wan, J. Wang, G. Yang, X. Zhang, Optimal siting of wind turbines using real-coded genetic algorithms, in: European Wind Energy Association Conference and Exhibition, Marseille, France, 2009.

[4] C. Wan, J. Wang, G. Yang, X. Zhang, Optimal micro-siting of wind farms by particle swarm optimization, in: Advances in Swarm Intelligence. First International Conference, Beijing, China, 198-205, 2010.

[5] G. Marmidis, S. Lazarou, E. Pyrgioti, Optimal placement of wind turbines in a wind park using Monte Carlo simulation, Renewable Energy 33 (2008) $1455-1460$.

[6] C. Zhang, G. Hou, J. Wang, A fast algorithm based on the submodular property for optimization of wind turbine positioning, Renewable Energy 36 (2011) 2951-2958.

[7] N. O. Jensen, A note on wind generator interaction, Tech. Rep., Risø National Laboratory, DK-4000 Roskilde, Denmark, 1983.

[8] G. C. Larsen, A simple wake calculation procedure, Tech. Rep., Ris $\varnothing$ National Laboratory, DK-4000 Roskilde, Denmark, URL http://www.risoe.dk/rispubl/VEA/veapdf/ris-m-2760.pdf, 1988. 
[9] T. Ishihara, A. Yamaguchi, Y. Fujino, Development of a new wake model based on a wind tunnel experiment, URL http://windeng.t.u-tokyo.ac.jp/posters/2004_gwp_poster.pdf, 2004 .

[10] P. K. Chaviaropoulos, M. O. L. Hansen, Investigating three-dimensional and rotational effects on wind turbine blades by means of a quasi-3D Navier-Stokes solver, Journal of Fluids Engineering 122 (2000) 330-336.

[11] A. Jimenez, A. Crespo, E. Migoya, J. Garcia, Advances in large-eddy simulation of a wind turbine wake, Journal of Physics: Conference Series 75 (012041).

[12] F. Zahle, N. N. Sørensen, On the influence of far-wake resolution on wind turbine flow simulations, Journal of Physics: Conference Series 75 (012042).

[13] Y.-T. Wu, F. Porté-Agel, Large-eddy simulation of wind-turbine wakes: evaluation of turbine parametrisations, Boundary-Layer Meteorol 138 (2011) 345-366.

[14] X. Yang, F. Sotiropoulos, On the predictive capabilities of LES-actuator disk model in simulating turbulence past wind turbines and farms, in: American Control Conference, 2878-2883, 2013.

[15] L. P. Chamorro, R. E. A. Arndt, F. Sotiropoulos, Turbulent flow properties around a staggered wind farm, Boundary-Layer Meteorol 141 (2011) 349367.

[16] W. Tian, A. Ozbay, H. Hu, An experimental study on the effects of incoming wind conditions on wind turbine aerpmechanics, in: 6th International Symposium on Fluid Machinery and Fluid Engineering, 1-8, 2014.

[17] M. X. Song, K. Chen, Z. Y. He, X. Zhang, Wake flow model of wind turbine using particle simulation, Renewable Energy 41 (2012) 185-190.

[18] M. X. Song, K. Chen, Z. Y. He, X. Zhang, Bionic optimization for micrositing of wind farm on complex terrain, Renewable Energy 50 (2013) 551557.

[19] M. X. Song, K. Chen, Z. Y. He, X. Zhang, Optimization of wind farm micro-siting for complex terrain using greedy algorithm, Energy 67 (2014) 454-459.

[20] M. X. Song, K. Chen, X. Zhang, J. Wang, The lazy greedy algorithm for power optimization of wind turbine positioning on complex terrain, Energy $80(2015)$ 567-574.

[21] I. Katic, J. Højstrup, N. O. Jensen, A simple model for cluster efficiency, in: European Wind Energy Association Conference and Exhibition, Rome, Italy, 407-410, 1986. 
[22] J. S. González, A. G. G. Rodriguez, J. C. Mora, J. R. Santos, M. B. Payan, Optimization of wind farm turbines layout using an evolutive algorithm, Renewable Energy 35 (2010) 1671-1681.

[23] K. Chen, M. Song, X. Zhang, The random walking method for the steady linear convection-diffusion equation with axisymmetric disc boundary, Science China Technological Sciences 57 (4) (2014) 804-810.

[24] G. E. P. Box, M. E. Muller, A note on the generation of random normal deviates, Annals of Mathematical Statistics 29 (2) (1958) 610-611.

[25] A. Einstein, Investigations of the theory of Brownian movement, Dover Publications, Inc, 1965.

[26] G. J. Taylor, D. J. Milborrow, D. N. McIntosh, D. T. Swift-Hook, Wake measurements on the Nibe windmills, in: Seventh BWEA Wind Energy Conference, 67-73, 1985.

[27] C. Croonenbroeck, C. M. Dahl, Accurate medium-term wind power forecasting in a censored classification framework, Energy 73 (2014) 221-232.

[28] J. M. L. M. Palma, F. A. Castro, L. F. Ribeiro, A. H. Rodrigues, A. P. Pinto, Linear and nonlinear models in wind resource assessment and wind turbine micro-siting in complex terrain, Journal of Wind Engineering and Industrial Aerodynamics 96 (2008) 2308-2326. 


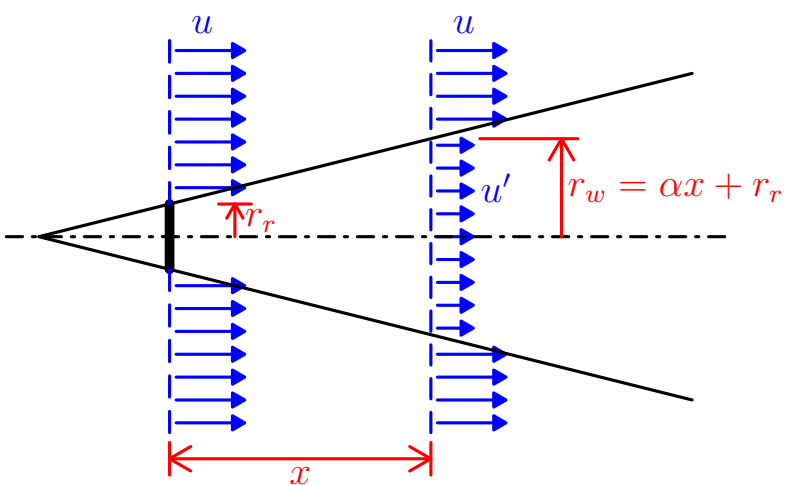

Figure 1: Schematic of the linear wake model.

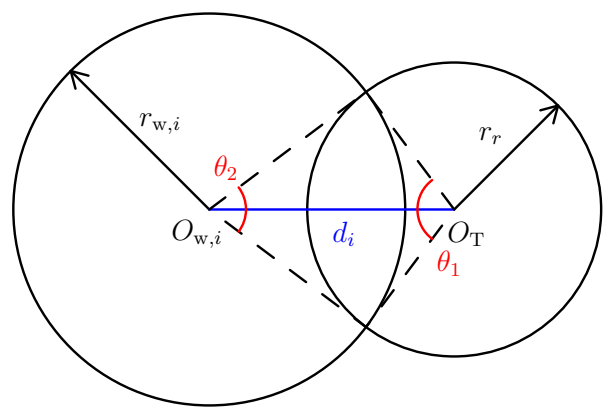

Figure 2: Overlapped area of the wake region and the turbine being affected

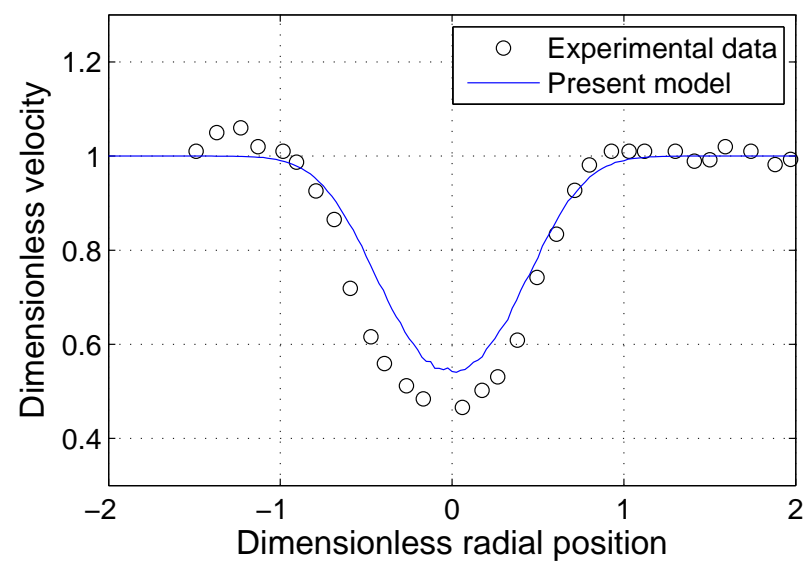

Figure 3: Velocity at $X=2.5 D$ for the single turbine case. 


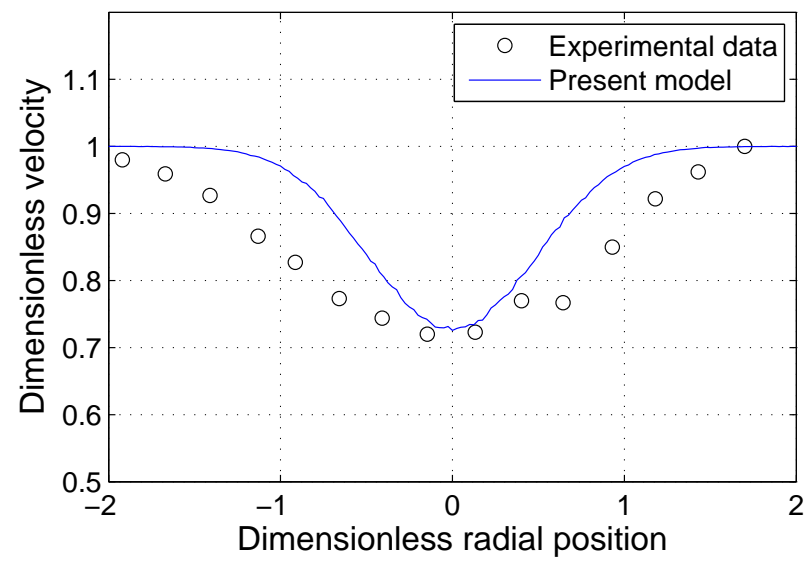

Figure 4: Velocity at $X=6 D$ for the single turbine case.

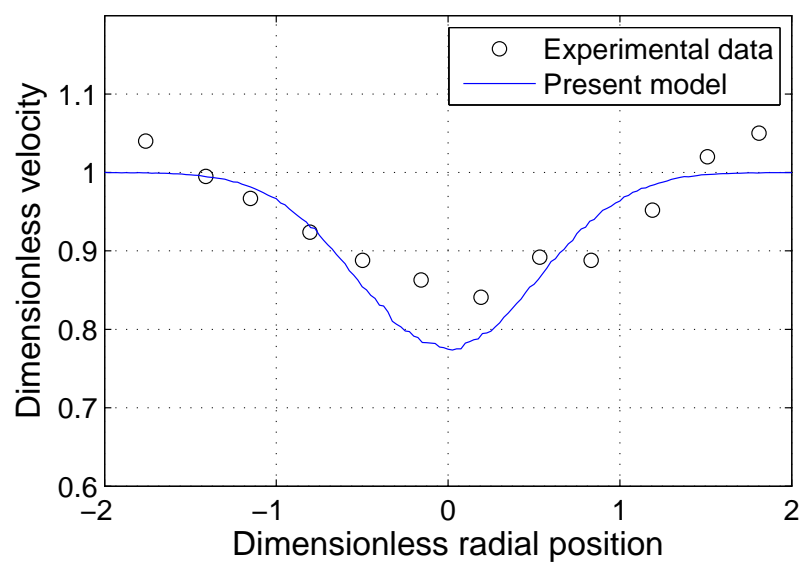

Figure 5: Velocity at $X=7.5 \mathrm{D}$ for the single turbine case.

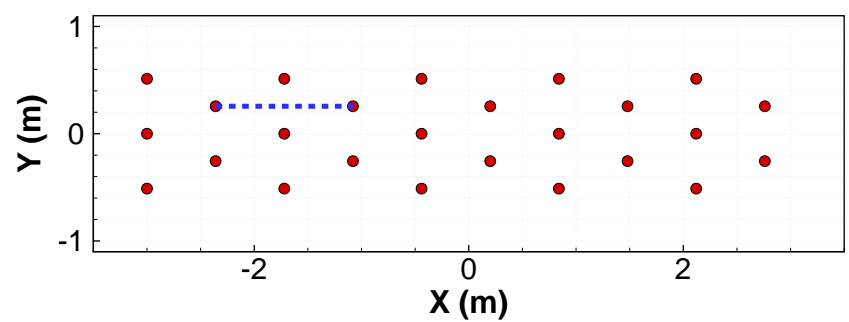

Figure 6: Positions of the miniature turbines in a wind tunnel. 


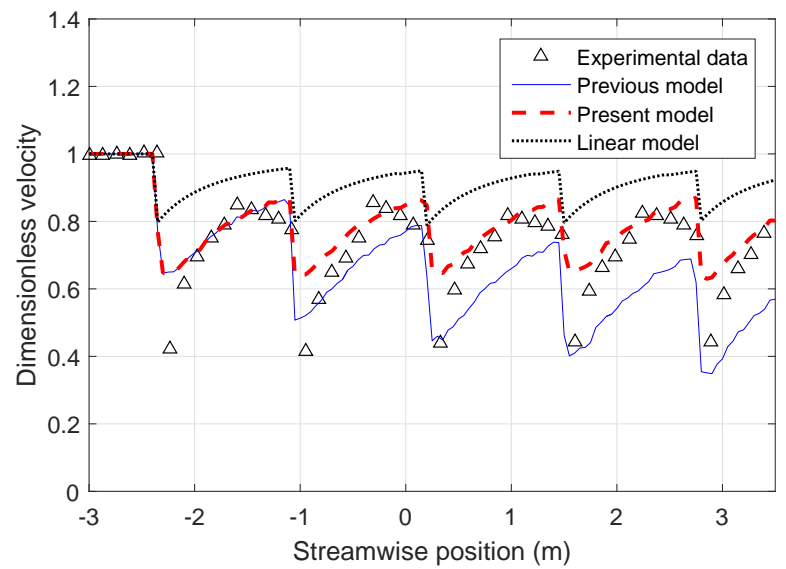

Figure 7: Velocity along the streamwise direction at the height of the hub $(Z=0.104 \mathrm{~m})$ for the multiple turbines case.

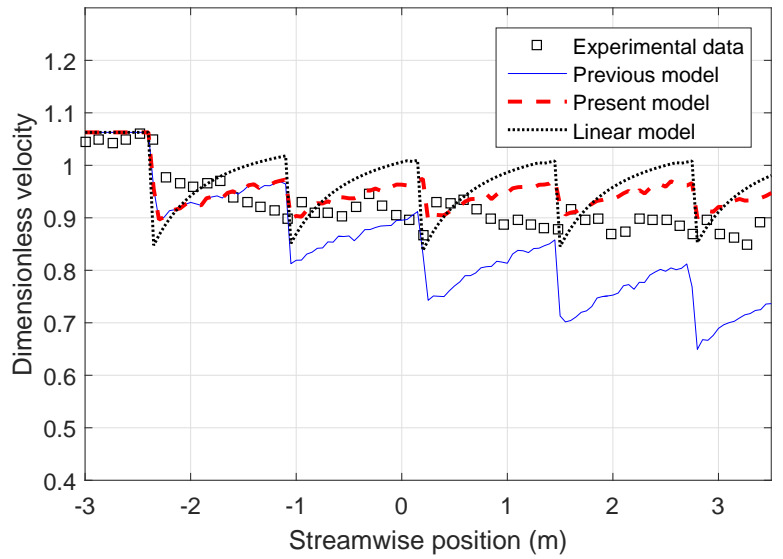

Figure 8: Velocity along the streamwise direction at rotor top $(Z=0.168 \mathrm{~m})$ for the multiple turbines case. 


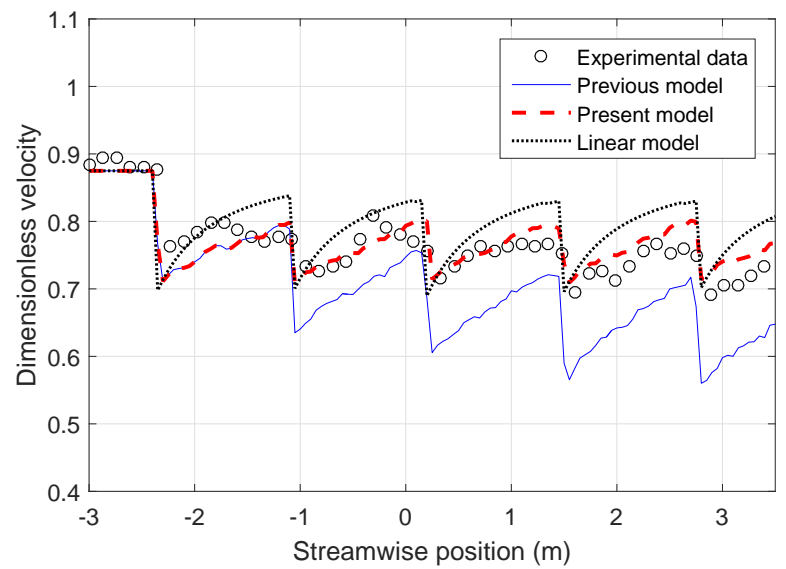

Figure 9: Velocity along the streamwise direction at the rotor bottom $(Z=0.040 \mathrm{~m})$ for the multiple turbines case.

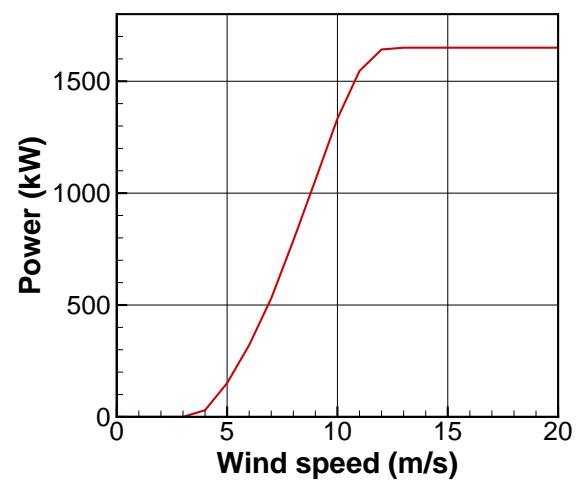

Figure 10: Power curve of the Vestas V82 wind turbine. 


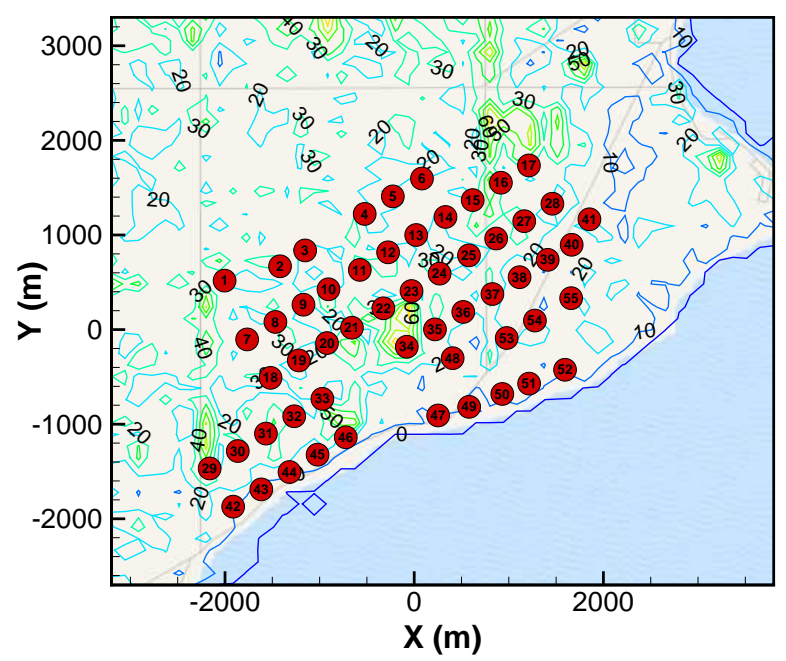

Figure 11: Altitude contours and turbine positions of the Wattle Point wind farm.

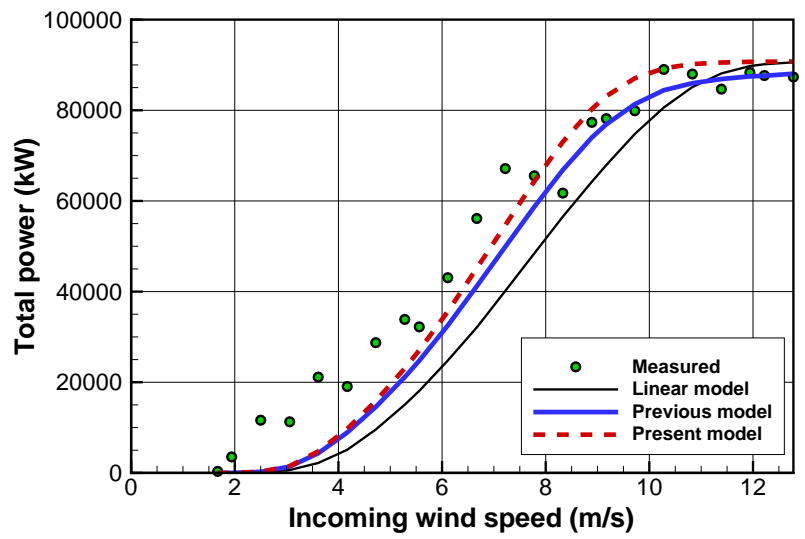

Figure 12: Total power of the Wattle Point wind farm under WSW wind direction. 


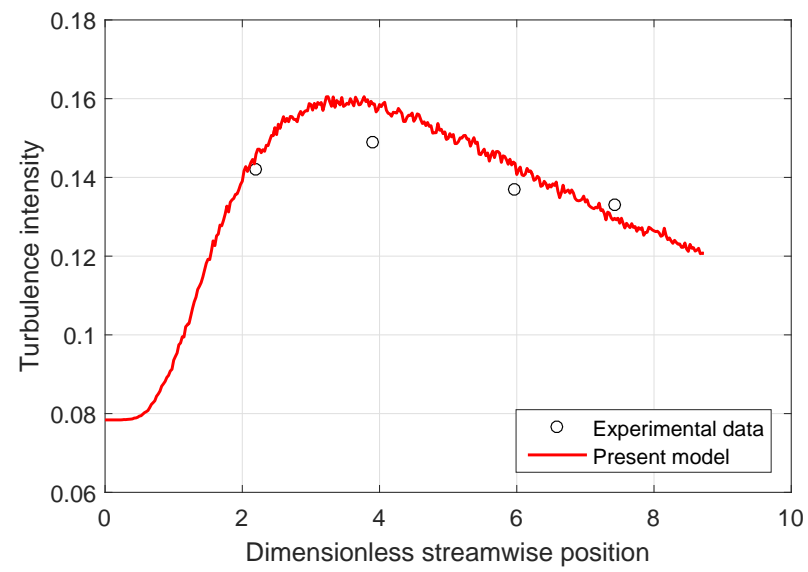

Figure 13: Turbulence along the axis of the turbine for the single turbine case.

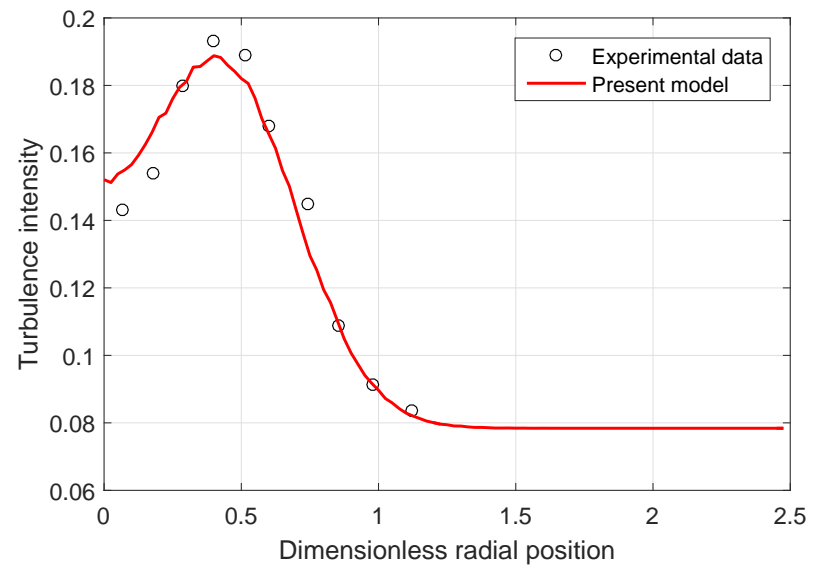

Figure 14: Turbulence at $X=2.5 D$ for the single turbine case. 


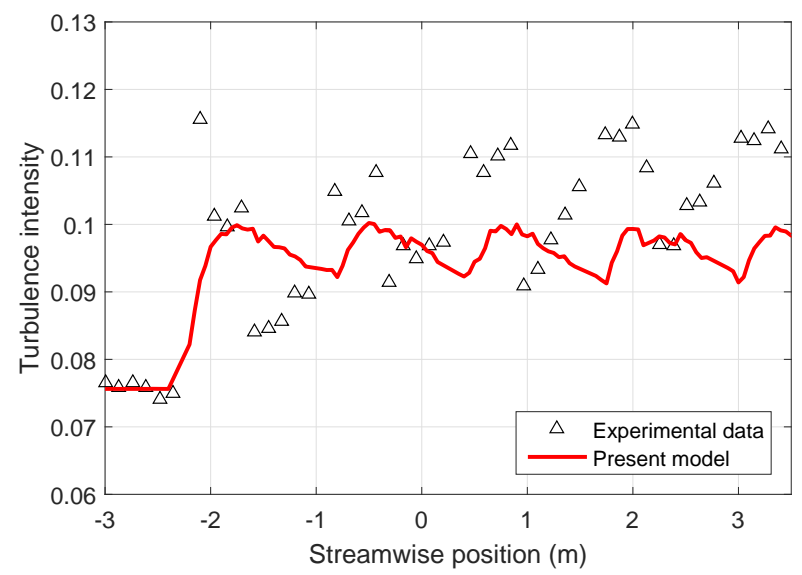

Figure 15: Turbulence along the streamwise direction at the height of the hub $(Z=0.104 \mathrm{~m})$ for the multiple turbines case.

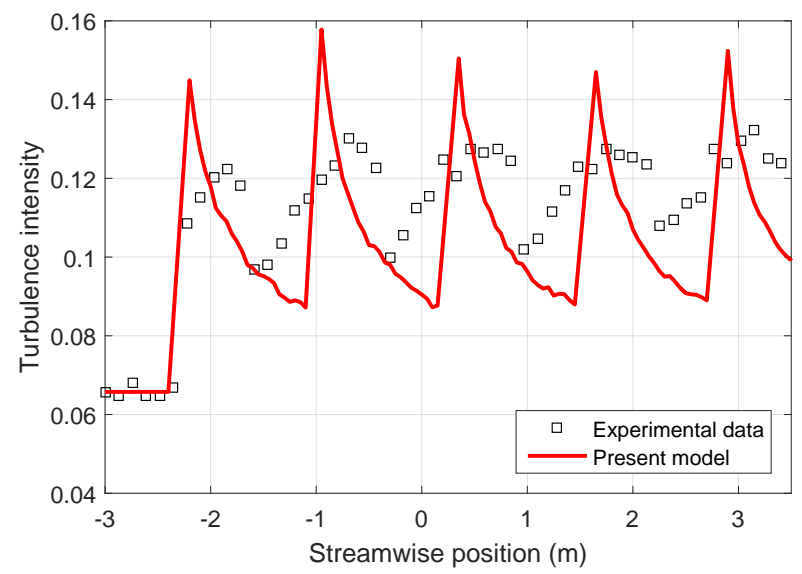

Figure 16: Turbulence along the streamwise direction at rotor top $(Z=0.168 \mathrm{~m})$ for the multiple turbines case. 


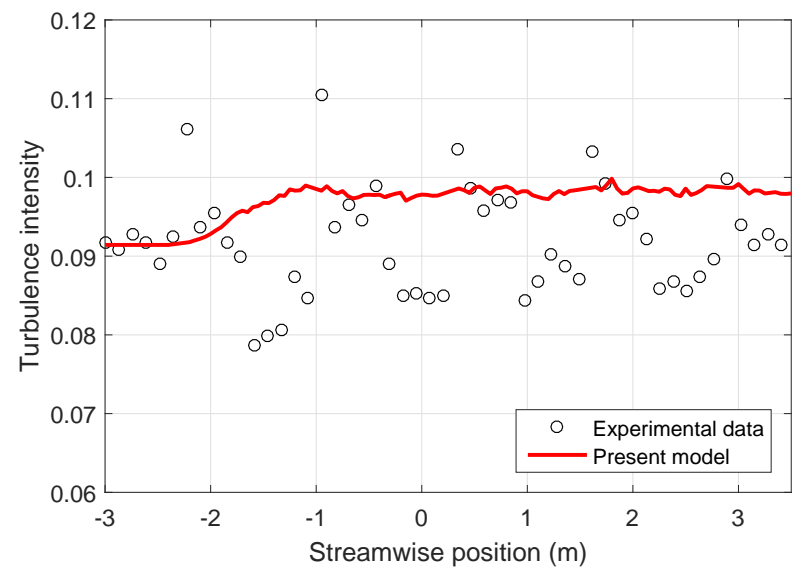

Figure 17: Turbulence along the streamwise direction at the rotor bottom $(Z=0.040 \mathrm{~m})$ for the multiple turbines case.

Table 1: Averaged velocity discrepancies between the result calculated by the present model and the experimental data within the region marked by blue dashed line.

\begin{tabular}{cc}
\hline$C_{\mathrm{T}}$ & Discrepancy \\
\hline 0.40 & $6.4026 \%$ \\
0.50 & $4.9895 \%$ \\
0.58 & $4.6903 \%$ \\
0.59 & $4.6674 \%$ \\
0.60 & $4.7091 \%$ \\
0.61 & $4.7755 \%$ \\
0.70 & $6.2361 \%$ \\
\hline
\end{tabular}

Table 2: Mean absolute errors of the evaluated dimensionless velocity for the multiple turbines case.

\begin{tabular}{cccc}
\hline & Hub & Top & Bottom \\
\hline Previous model & 0.096 & 0.082 & 0.064 \\
Present model & 0.059 & 0.043 & 0.022 \\
Linear model & 0.172 & 0.060 & 0.040 \\
\hline
\end{tabular}

Table 3: Root mean square errors of the evaluated dimensionless velocity for the multiple turbines case.

\begin{tabular}{cccc}
\hline & Hub & Top & Bottom \\
\hline Previous model & 0.120 & 0.101 & 0.073 \\
Present model & 0.085 & 0.050 & 0.028 \\
Linear model & 0.200 & 0.072 & 0.048 \\
\hline
\end{tabular}


Table 4: Minimum discrepancies of turbulence intensity between the simulated results and the experimental data for determination of $\tau$.

\begin{tabular}{cc}
\hline$\tau$ & Discrepancy \\
\hline 10 & 0.009657 \\
20 & 0.006243 \\
30 & 0.005543 \\
31 & 0.004664 \\
32 & 0.005650 \\
40 & 0.006814 \\
50 & 0.007086 \\
\hline
\end{tabular}

Table 5: Turbulent intensities and evaluated errors for the multiple turbines case.

\begin{tabular}{cccc}
\hline & Hub & Top & Bottom \\
\hline Measured incoming flow & 0.076 & 0.066 & 0.091 \\
Measured average in wake & 0.102 & 0.118 & 0.092 \\
Evaluated average error & 0.0080 & 0.0147 & 0.0070 \\
Relative error & $7.8 \%$ & $12.5 \%$ & $7.6 \%$ \\
\hline
\end{tabular}

